\begin{tabular}{|c|l|}
\hline Title & $\begin{array}{l}\text { Generalized theoretical method for the interaction between arbitrary nonuniform electric field and molecular vibrations: } \\
\text { Toward near-field infrared spectroscopy and microscopy }\end{array}$ \\
\hline Author(s) & Iwasa, Takeshi; Takenaka, Masato; Taketsugu, Tetsuya \\
\hline Citation & $\begin{array}{l}\text { Journal of chemical physics, 144(12), 124116 } \\
\text { https://doi.org/10.1063/1.4944937 }\end{array}$ \\
\hline Issue Date & $2016-03$-29 \\
\hline Doc URL & http://hdl.handle.net/2115/64894 \\
\hline Rights & $\begin{array}{l}\text { The following article has been accepted by Journal of chemical physics. A fter it is published, it will be found at } \\
10.1063 / 1.4944937 .\end{array}$ \\
\hline Type & article \\
\hline File Information & \begin{tabular}{l}
$1.4944937 . p d f$ \\
\hline
\end{tabular} \\
\hline
\end{tabular}

Instructions for use 


\title{
Generalized theoretical method for the interaction between arbitrary nonuniform electric field and molecular vibrations: Toward near-field infrared spectroscopy and microscopy
}

\author{
Takeshi Iwasa, ${ }^{\text {a) }}$ Masato Takenaka, and Tetsuya Taketsugu \\ Department of Chemistry, Faculty of Science, Hokkaido University, Sapporo 060-0810, Japan
}

(Received 4 January 2016; accepted 16 March 2016; published online 31 March 2016)

\begin{abstract}
A theoretical method to compute infrared absorption spectra when a molecule is interacting with an arbitrary nonuniform electric field such as near-fields is developed and numerically applied to simple model systems. The method is based on the multipolar Hamiltonian where the light-matter interaction is described by a spatial integral of the inner product of the molecular polarization and applied electric field. The computation scheme is developed under the harmonic approximation for the molecular vibrations and the framework of modern electronic structure calculations such as the density functional theory. Infrared reflection absorption and near-field infrared absorption are considered as model systems. The obtained IR spectra successfully reflect the spatial structure of the applied electric field and corresponding vibrational modes, demonstrating applicability of the present method to analyze modern nanovibrational spectroscopy using near-fields. The present method can use arbitral electric fields and thus can integrate two fields such as computational chemistry and electromagnetics. @ 2016 AIP Publishing LLC. [http://dx.doi.org/10.1063/1.4944937]
\end{abstract}

\section{INTRODUCTION}

Vibrational spectroscopy has been a powerful tool to gain structural information of molecules. ${ }^{1}$ Recent development of vibrational spectroscopy extends to other fields including surface science, nano-optics, or biosciences. ${ }^{2-4}$ For some cases, applied electric fields for molecules are spatially anisotropic or nonuniform. Electric fields in the infraredreflection absorption spectroscopy (IR-RAS) are anisotropic and polarized in the surface normal direction, with which molecular orientations can be studied in addition to molecular structures adsorbed on surface. ${ }^{5}$ Attenuated total reflection (ATR) spectroscopy takes advantage of the evanescent field whose intensity decreases exponentially away from the surface $^{6-8}$ and, although in the case of atomic spectroscopy, the quadrupole response was observed. ${ }^{9,10}$ In addition to this, surface plasmon has recently been widely adopted to enhance spectroscopic signals. Among others, surfaceenhanced Raman spectroscopy (SERS) would be the most popular technique that takes advantage of the electric nearfields of surface plasmon in the close vicinity of metal nanostructures. ${ }^{11}$ The use of near-fields allows us to study molecules at the nanometer scale beyond the diffraction limit, and ultimately at the single molecule level. ${ }^{12}$ In ATR measurements using IR, surface plasmon of metal nanoparticles is used to enhance the field strength and hence the IR signal, which is called surface enhanced IR absorption spectroscopy (SEIRAS). ${ }^{13,14}$ Also known is surface enhanced IR scattering (SEIRS) that adopts designed nanostructure as an antenna for plasmon enhancement and the Fano resonance in the IR measurements. ${ }^{15,16}$ In addition to

a)tiwasa@mail.sci.hokudai.ac.jp these developments, scanning near-field infrared microscopy (SNIM) adopting plasmonic near-fields has been invented and demonstrated its ability for nanometer scale spatial resolution beyond the diffraction limit, ${ }^{17-21}$ as in tip-enhanced Raman spectroscopy achieving very high resolution down to a single-molecule level. ${ }^{22,23}$ In these nanospectroscopic or microscopic measurements, the plasmonic near-fields may have complicated spatial distributions over target molecules requiring proper understandings on the near-field and the interaction between these near-fields and molecular vibrations.

The near-fields around nanostructures have mainly been studied by solving the Maxwell equations with various theoretical techniques including the discrete dipole approximation, finite difference time-domain method, finiteelement methods, and Green-function methods. ${ }^{24}$ On the other hand, the light-matter interaction for molecular vibrations has been studied under the dipole approximation because the wavelength of light in IR or even in visible region is much larger than a molecule. However, the effect of the field gradient has been known for surface enhanced Raman spectroscopy. ${ }^{25}$ Recent studies including the nano-optical measurements where molecules are interacting with a highly localized electric field also require theoretical methods beyond the dipole approximation. ${ }^{26-29}$ These studies suggest needs for more general theoretical framework, but still practically tractable with moderate computational cost.

Apart from the vibrational spectroscopy, theoretical frameworks of optical response taking account of the full nonuniform and self-consistent light-matter interactions have so far been developed for, mostly, electronic responses ${ }^{30-33}$ and demonstrated the importance of the full light-matter interactions in optical response for various nanosystems such as nanocrystals, semiconductor quantum dots, nanoparticles, 
and molecular compounds. ${ }^{34-41}$ On the other hand, multipole effects concerning the nonuniform light-matter interaction (i.e., beyond the dipole approximation) were discussed in nanoparticles ${ }^{37,39}$ and molecular compounds. ${ }^{36}$ Also recent studies clarifies the field gradient intrinsically causes the non-linear responses. ${ }^{42}$

Concerning the vibrational spectroscopy, the mechanism of SERS has extensively been studied, in particular, in the field of molecular science with main focus on the mechanisms of surface enhancements. ${ }^{35,43,44}$ However, corresponding theoretical studies for the infrared absorption have been scarce, in particular, studies focusing on the effects of spatial structures of near-fields, and theoretical tools still need to be developed. In this study, we propose a theoretical method for infrared absorption spectroscopy with near-field on the basis of the multipolar Hamiltonian. This method is an extension of our previous study for nonuniform optical responses of electron dynamics ${ }^{45}$ and IRRAS study. ${ }^{46}$

\section{THEORY}

\section{A. IR absorption based on the multipolar Hamiltonian}

In the multipolar Hamiltonian, the interaction between molecule and electric fields is given by the following equation: ${ }^{45,47-49}$

$$
V_{\text {int }}=\int \boldsymbol{P}(\boldsymbol{r}) \cdot \boldsymbol{E}(\boldsymbol{r}) d \boldsymbol{r},
$$

where $\boldsymbol{P}$ and $\boldsymbol{E}$ are the polarization and the external electric fields, respectively, and both of them depend on the spatial coordinate $\boldsymbol{r}$. Under small vibrational amplitudes, the linear term of a power series of the Hamiltonian in the normal coordinate should be much larger than the higher terms, as in the conventional IR absorption. ${ }^{1}$ The linear term of the expansion of Eq. (1) is expressed as

$$
V_{\text {int }}^{k}=Q_{k} \int\left(\partial \boldsymbol{P}(\boldsymbol{r}) / \partial Q_{k}\right) \cdot \boldsymbol{E}(\boldsymbol{r}) d \boldsymbol{r} .
$$

This is a general form of the interaction between molecular vibrations and arbitral electric field. When the electric field is uniform, the so-called dipole approximation can be obtained by expanding the polarization around the molecular center and taking the first term, where the spatial variation of the electric field is totally neglected.

Let us consider the integration first and then take the derivative by the normal coordinate,

$$
\begin{aligned}
V_{\text {int }}^{k} & =Q_{k} \frac{\partial}{\partial Q_{k}} \int \boldsymbol{P}(\boldsymbol{r}) \cdot \boldsymbol{E}(\boldsymbol{r}) d \boldsymbol{r} \\
& \equiv Q_{k} \frac{\partial V_{\text {int }}}{\partial Q_{k}} .
\end{aligned}
$$

By taking the expectation value using electronic and vibrational wave functions such as $\left|\Psi_{n}\right\rangle\left|v_{n}\right\rangle$, we can obtain the IR intensity. The electronic state is assumed to be the ground state, $\left|\Psi_{0}\right\rangle$, and vibrational wave functions are obtained under the harmonic potential around the equilibrium structure as the conventional IR spectroscopy. With these assumptions, the IR absorption intensity from the vibrational ground state to the first excited state, $\left|v_{0}\right\rangle \rightarrow\left|v_{1}\right\rangle$, is proportional to the following value:

$$
\begin{aligned}
& \left\langle v_{1}\left|\left\langle\Psi_{0}\left|V_{\text {int }}^{k}\right| \Psi_{0}\right\rangle\right| v_{0}\right\rangle \\
& =\left\langle v_{1}\left|Q_{k}\right| v_{0}\right\rangle \frac{\partial\left\langle\Psi_{0}\left|V_{\text {int }}\right| \Psi_{0}\right\rangle}{\partial Q_{k}} \sim \frac{\partial\left\langle\Psi_{0}\left|V_{\text {int }}\right| \Psi_{0}\right\rangle}{\partial Q_{k}} \equiv \frac{\partial A}{\partial Q_{k}},
\end{aligned}
$$

where $\left\langle v_{1}\left|Q_{k}\right| v_{0}\right\rangle$ is a constant. The generalized IR absorption intensity $I_{k}$ is obtained by the square of the absolute value of the normal coordinate derivative of $A$, as in the conventional IR intensity is proportional to those of the dipole moment, instead of $A$, under the so-called dipole and harmonic approximations,

$$
I_{k} \propto\left|\frac{\partial A}{\partial Q_{k}}\right|^{2} .
$$

The normal coordinate derivative $\partial A / \partial Q_{k}$ is obtained using the transformation matrix $\boldsymbol{L}$ between the normal coordinate $Q_{k}$ and the Cartesian atomic displacements $b_{i}$ $(i=1 \cdots 3 N)$, and here $N$ is the number of atoms in a molecule,

$$
\frac{\partial A}{\partial Q_{k}}=\sum_{i=1}^{3 N} L_{i, k} \frac{\partial A}{\partial b_{i}}
$$

\section{B. Calculation of $\boldsymbol{A}$ using polarization and electron density difference}

To calculate $A$, the inner product of the polarization and electric field should be integrated. The exact form of the polarization operator for a molecule is given by ${ }^{47-49}$

$$
\hat{\boldsymbol{P}}(\boldsymbol{r})=\sum_{\alpha} e_{\alpha}\left(\hat{\boldsymbol{q}}_{\alpha}-\boldsymbol{R}\right) \int_{0}^{1} d \lambda \delta\left(\boldsymbol{r}-\boldsymbol{R}-\lambda\left(\hat{\boldsymbol{q}}_{\alpha}-\boldsymbol{R}\right)\right),
$$

where $e_{\alpha}$ and $\hat{\boldsymbol{q}}_{\alpha}$ are the charge and position of $\alpha$ th particle such as an electron or nucleus. $\boldsymbol{R}$ is the molecular center of mass. By using Eq. (7), the expectation value of $A$ is expressed as

$$
\begin{aligned}
A\left(\left\{\boldsymbol{R}_{\alpha}\right\}\right)= & \int d \boldsymbol{r}\langle\hat{\boldsymbol{P}}(\boldsymbol{r})\rangle \cdot \boldsymbol{E}(\boldsymbol{r}) \\
= & \int d \boldsymbol{r}\left\langle\hat{\boldsymbol{P}}_{\mathrm{elec}}(\boldsymbol{r})\right\rangle \cdot \boldsymbol{E}(\boldsymbol{r})+\int d \boldsymbol{r}\left\langle\hat{\boldsymbol{P}}_{\mathrm{N}}(\boldsymbol{r})\right\rangle \cdot \boldsymbol{E}(\boldsymbol{r}) \\
= & -\int e \rho(\boldsymbol{r})(\boldsymbol{r}-\boldsymbol{R}) \cdot \boldsymbol{E}_{\mathrm{eff}}(\boldsymbol{r}) d \boldsymbol{r} \\
& +\sum_{\alpha} Z_{\alpha}\left(\boldsymbol{R}_{\alpha}-\boldsymbol{R}\right) \cdot \boldsymbol{E}_{\mathrm{eff}}\left(\boldsymbol{R}_{\alpha}\right),
\end{aligned}
$$

where $\hat{\boldsymbol{P}}_{\text {elec/N }}$ is the polarization operator for electrons or ions, respectively, $\rho(\boldsymbol{r})$ is the electron density, and $Z_{\alpha}$ and $\boldsymbol{R}_{\alpha}$ are the charge and position of $\alpha$ th nucleus. We stress that $A$ depends on the molecular structure, i.e., the set of atomic coordinates $\left\{\boldsymbol{R}_{\alpha}\right\}$. The derivation for $\left\langle\hat{\boldsymbol{P}}_{\text {elec } / \mathrm{N}}\right\rangle$ is given in the Appendix. The effective electric field $\boldsymbol{E}_{\text {eff }}$ is defined as follows: 45

$$
\boldsymbol{E}_{\mathrm{eff}}(\boldsymbol{r} ; \boldsymbol{R})=\int_{0}^{1} d \lambda \boldsymbol{E}(\boldsymbol{R}+\lambda(\boldsymbol{r}-\boldsymbol{R})) .
$$

Equation (8) can be simplified further by considering the electron density difference defined by

$$
\delta \rho(\boldsymbol{r}) \equiv \rho(\boldsymbol{r})-\rho^{\mathrm{atom}}(\boldsymbol{r}),
$$


where $e=1$ is assumed for simplicity and $\rho^{\text {atom }}$ is the sum of the electron densities of the neutral atoms in a molecule, which compensates the nuclear charges of all the ions in the system, since any aggregates of neutral atoms show no polarizations,

$$
\rho^{\mathrm{atom}}(\boldsymbol{r})=\sum_{\alpha} Z_{\alpha} \delta\left(\boldsymbol{r}-\boldsymbol{R}_{\alpha}\right)
$$

By using this electron density difference, $A$ becomes

$$
\begin{aligned}
A & =-\int e \rho(\boldsymbol{r})(\boldsymbol{r}-\boldsymbol{R}) \cdot \boldsymbol{E}_{\mathrm{eff}}(\boldsymbol{r}) d \boldsymbol{r}+\sum_{\alpha} Z_{\alpha}\left(\boldsymbol{R}_{\alpha}-\boldsymbol{R}\right) \cdot \boldsymbol{E}_{\mathrm{eff}}\left(\boldsymbol{R}_{\alpha}\right) \\
& =\int d \boldsymbol{r}\left[-e \rho(\boldsymbol{r})(\boldsymbol{r}-\boldsymbol{R})+\sum_{\alpha} Z_{\alpha}(\boldsymbol{r}-\boldsymbol{R}) \delta\left(\boldsymbol{r}-\boldsymbol{R}_{\alpha}\right)\right] \cdot \boldsymbol{E}_{\mathrm{eff}}(\boldsymbol{r}) \\
& =\int d \boldsymbol{r}\left[-e \rho(\boldsymbol{r})+\sum_{\alpha} Z_{\alpha} \delta\left(\boldsymbol{r}-\boldsymbol{R}_{\alpha}\right)\right](\boldsymbol{r}-\boldsymbol{R}) \cdot \boldsymbol{E}_{\mathrm{eff}}(\boldsymbol{r}) \\
& =-\int \delta \rho(\boldsymbol{r})(\boldsymbol{r}-\boldsymbol{R}) \cdot \boldsymbol{E}_{\mathrm{eff}}(\boldsymbol{r}) d \boldsymbol{r} .
\end{aligned}
$$

This form can be seen as such that a point dipole at each grid interacts with the effective electric field, defined by Eq. (9), which now contains all the effects of the molecular multipoles. By its definition, $\boldsymbol{E}_{\text {eff }}$ can be understood as an average electric field between point $\boldsymbol{r}$ and molecular center $\boldsymbol{R}$. In other words, in the present formulation, IR spectra calculated from $A$ do not depend on the origin but depend on the center of molecule.

\section{Relation to the multipole expansion}

Before moving to the computational details, let us mention about the relation between the present formulation and conventional multipole expansion approaches, starting from the dipole approximation followed by step by step inclusions of higher multipoles. Eq. (7) can be expanded in a Taylor series leading to the dipole, quadrupole, octapole, and higher-order multipole terms. Thus by expanding $\boldsymbol{P}$ in Eq. (1) according to Eq. (7) and integrating the resulting equation with respect to $\lambda$, we obtain

$$
\begin{aligned}
\int d \boldsymbol{r} \hat{\boldsymbol{P}}(\boldsymbol{r}) \cdot \boldsymbol{E}(\boldsymbol{r}, t)= & \sum_{i}\left(\sum_{\alpha} e_{\alpha}\left(\hat{\boldsymbol{q}}_{\alpha}-\boldsymbol{R}\right)_{i}\right) \cdot E_{i}(\boldsymbol{R}, t)-\sum_{i, j}\left(\frac{1}{2 !} \sum_{\alpha} e_{\alpha}\left(\hat{\boldsymbol{q}}_{\alpha}-\boldsymbol{R}\right)_{i}\left(\hat{\boldsymbol{q}}_{\alpha}-\boldsymbol{R}\right)_{j}\right) \nabla_{i} E_{j}(\boldsymbol{R}, t) \\
& +\sum_{i, j, k}\left(\frac{1}{3 !} \sum_{\alpha} e_{\alpha}\left(\hat{\boldsymbol{q}}_{\alpha}-\boldsymbol{R}\right)_{i}\left(\hat{\boldsymbol{q}}_{\alpha}-\boldsymbol{R}\right)_{j}\left(\hat{\boldsymbol{q}}_{\alpha}-\boldsymbol{R}\right)_{k}\right) \nabla_{i} \nabla_{j} E_{k}(\boldsymbol{R}, t)+\cdots \\
\equiv & \sum_{i} \hat{\mu}_{i} E_{i}+\sum_{i, j} \hat{Q}_{i j} \nabla_{i} E_{j}+\sum_{i, j, k} \hat{O}_{i j k} \nabla_{i} \nabla_{j} E_{k}+\cdots
\end{aligned}
$$

where $\nabla_{i}, \hat{\mu}_{i}, \hat{Q}_{i j}$, and $\hat{O}_{i j k}$ represent the operators of gradient, dipole, quadrupole, and octapole moments of a molecule, respectively, and the indices denote their $(x, y, z)$ tensorial components. These moments are defined at the molecular center $\boldsymbol{R}$. If an electric field varies slowly over a molecule, the light-matter interaction can be systematically improved by including higher multipole terms in this expansion, starting from the dipole interaction. However, when the field gradient is much sharp, the appropriate number of multipoles, or even dominant multipoles contributing to the light-matter interaction, becomes unclear and might depend on the system considered. Therefore, we develop more general approach by using the original form of the polarization operator (Eq. (7)) as is, without performing the Taylor expansion of the polarization. The use of the original polarization operator corresponds to the inclusion of the infinite number of multipole terms in the light-matter interaction.

\section{COMPUTATIONAL DETAILS}

Computational scheme for the above formulation is as follows. First, we perform the geometry optimization and normal mode analysis and obtain the electron density difference $\delta \rho$ as a grid data. Second, the effective electric field $\boldsymbol{E}_{\text {eff }}$ is calculated on the same grid as $\delta \rho$. Third using

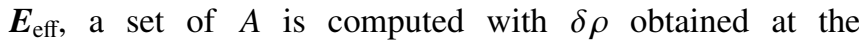
equilibrium and distorted geometries, for the latter all the atomic coordinates are slightly displaced in every directions. Fourth, the derivatives of $A$ with respect to the atomic Cartesian coordinates are calculated and are transformed into the normal coordinate derivatives using the transformation matrix $\boldsymbol{L}$. Finally, IR absorption spectrum is obtained from the square of the absolute value of the normal coordinate derivatives of $A$.

Before moving into detail, it should be noted that the present computational scheme only requires the grid data of an electron density difference (or the electron density and atomic coordinates) and electric field and therefore any electronic structure codes can be used.

\section{A. DFT calculations for electron density difference and normal coordinates}

The geometric, electronic, and vibrational properties such as the optimized structures, electron density differences, and eigenvalue and transformation matrix for normal modes of target systems are computed using a density functional theory code, SIESTA, ${ }^{50}$ at the level of $\mathrm{PBE}^{51} / \mathrm{DZP}^{52}$ For all systems considered in this study, unit cell is taken as $20 \times 20 \times 20 \AA^{3}$ and only gamma point is sampled. As the first application, IR and IRRAS spectra for $\mathrm{NO}$ on $\mathrm{Cu}_{4}$ are 
calculated, where the mesh cutoff of $200 \mathrm{Ry}$ is used. As the second application, IR spectra of aniline interacting with near-field are calculated, where the mesh cutoff of $100 \mathrm{Ry}$ is used because this value is found to reasonably give the normal IR spectrum. After obtaining equilibrium geometry, each atomic coordinate is displaced in the positive direction from the equilibrium coordinates by 0.04 a.u. and then the electron density differences $\delta \rho$ are obtained by performing single point calculations at those shifted coordinates.

\section{B. Effective electric field}

Effective electric fields are calculated using Eq. (9) by numerical integration as follows:

$$
\boldsymbol{E}_{\mathrm{eff}}(\boldsymbol{r} ; \boldsymbol{R})=\sum_{i=0}^{M} \frac{1}{M} \boldsymbol{E}\left(\boldsymbol{R}+\frac{i}{M} \boldsymbol{x}\right),
$$

where a difference vector $\boldsymbol{x}=\boldsymbol{r}-\boldsymbol{R}$ is introduced. As can be seen from this form, the effective electric field is an average between $\boldsymbol{r}$ and the molecular center $\boldsymbol{R}$. The parameter $M$ is set to be a constant during the calculation, irrespective to the position, i.e., independent from $|\boldsymbol{x}|$. According to our test, $M=30$ is enough for the near-field and beyond this, the spectra look the same. $M$ could depend on the electric field employed and should be tested when changing the field. In the future, an electric field should be obtained through solving the Maxwell equations for some nanostructure using, for instance, finite-difference time-domain technique or others.

\section{Derivative of $\boldsymbol{A}$}

Having obtained the grid data for the electron density difference $\delta \rho$ and the effective electric field $\boldsymbol{E}_{\text {eff }}$, we can calculate $A$ using Eq. (12) by a simple numerical integration over spatial grids. The spatial grid for the integration is determined by the mesh cutoff using SIESTA.

The derivatives of $A$ with respect to the atomic displacements $b_{i}$ are numerically obtained by a finite difference as follows:

$$
\frac{\partial A}{\partial b_{i}}=\frac{A\left(R_{i}^{\mathrm{eq}}+b_{i}\right)-A\left(R_{i}^{\mathrm{eq}}\right)}{\left|b_{i}\right|},
$$

where $R_{i}^{\mathrm{eq}}$ represents the $i$ th atomic coordinate at the equilibrium geometry. This atomic coordinate derivative $\partial A / \partial b_{i}$ is then transformed into $\partial A / \partial Q_{k}$ by using Eq. (6).

\section{APPLICATIONS}

In the following, we will give two independent applications of the present approach. As the first example, IRRAS is considered, where a uniform electric field along the surface normal interacts with surface molecule. In this case, the dipole approximation is valid but due to the anisotropic electric field, most available quantum chemical packages cannot be used to calculate IRRAS. The second application adopts the near field of the dipole radiation, which is an extreme model for near-field around metal tip or metallic nanoparticles. Although this model is too simplified to represent molecules around a metallic tip, it gives us the essence of highly nonuniform electric field with ultra sharp field gradient, which requires us to include uncounted number of multipoles.

\section{A. IRRAS}

In IRRAS measurements, one needs to take account of the direction of electric field because the incoming light and reflection light at the metal surface interfere each other. ${ }^{5}$ The interference results in the disappearance in the electric field parallel to the surface and remaining electric field normal to the surface excites molecules that are adsorbed on the surface. This is depicted in Fig. 1(a). One of the authors previously studied IRRAS by projecting the normal coordinate derivative of the dipole moment to the surface normal vector. ${ }^{46}$ In the present study, on the other hand, we can obtain IRRAS spectra by computing $A$ with uniform electric field $\boldsymbol{E}$, resulting in the same effect, i.e., only the dipole moment interacts with the electric field.

A model system for a surface-adsorbed molecule is shown in Fig. 1(b), where a simple diatomic molecule, NO, is adsorbed onto $\mathrm{Cu}_{4}$ cluster. Figure 1(c) shows that this simple model system has two representative IR active vibrational modes that are parallel and normal to the surface at the wavenumber of 499 and $1578 \mathrm{~cm}^{-1}$, respectively, among other minor modes that include $\mathrm{Cu}$ motions in their normal coordinates. As is apparent in Figures 1(c)-1(e), the polarized electric fields along the directions of surface parallel $\left(\boldsymbol{E}^{\|}\right)$and surface normal $\left(\boldsymbol{E}^{\perp}\right)$, respectively, cause enhancements to the corresponding vibrational modes. The latter is corresponding to the IRRAS spectrum. ${ }^{53-55}$ This method is useful for more complex molecule having lower symmetry that makes theoretical analysis much more difficult.

\section{B. Near-field excitations}

Next, we consider a more complex situation, such as SNIM or SEIRA/SEIRS experiments, which are schema-
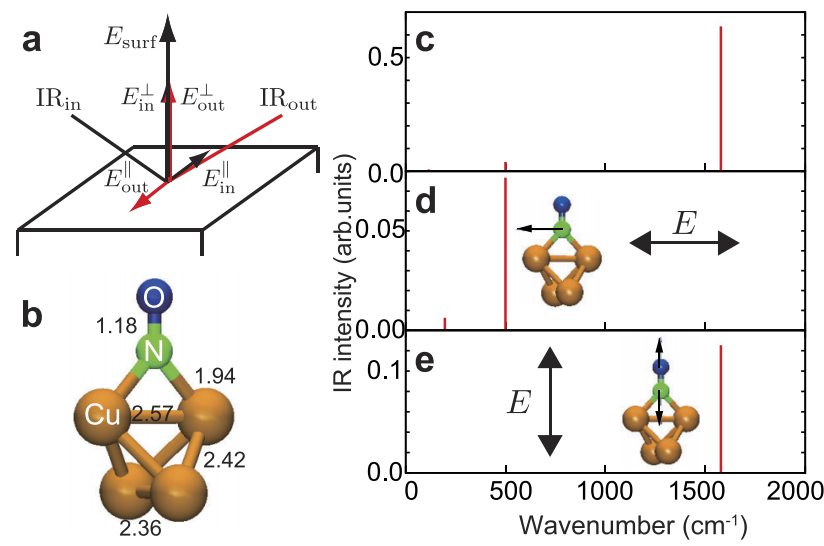

FIG. 1. (a) Schematic of IRRAS setup where the interferences of the electric fields of the incident ( $\operatorname{IR}_{\text {in }}$ : black line) and reflected light $\left(\mathrm{IR}_{\text {out }}\right.$ : red line) in the parallel $\left(E^{\|}\right)$and normal $\left(E^{\perp}\right)$ directions to the surface give rise to polarized electric field $\left(E_{\text {surf }}\right)$ in the direction of surface normal. (b) NO adsorbed onto $\mathrm{Cu}$ surface (modeled by $\mathrm{Cu}_{4}$ ) along with bond lengths (given in $\AA$ ). (c) The conventional IR spectra, i.e., without any polarization. The IR spectra obtained by uniform electric fields that are polarized in the direction of (d) surface parallel and (e) surface normal, in which insets show the corresponding vibrational modes. 
tically shown in Figs. 2(a) and 2(b). In these spectroscopic measurements, molecules are excited by the infrared light localized around nanostructures, and the localized light has sharp intensity gradients and nonuniform spatial distributions in its electric field line. In this study, we use a simple but rather fictitious model for the near-field. The calculation of realistic near-field as is widely done in the field of optics will be combined in the future for more realistic situations and then direct comparisons with experiments will be possible. As a test molecule, aniline is chosen because this is a very simple molecule having two different end groups along the molecular principal axis, this has the mirror symmetry letting the present discussion simple, and this molecule was studied in our previous study. ${ }^{46}$

\section{Near-field}

In this study, we approximate the electric field around the tip by the near-part of the dipole radiation field, the analytic form of which is given as ${ }^{56}$

$$
\boldsymbol{E}_{\mathrm{dip}}(\boldsymbol{r}) e^{-i k r}=\frac{[3 \boldsymbol{n}(\boldsymbol{n} \cdot \boldsymbol{\mu})-\boldsymbol{\mu}]}{4 \pi \varepsilon_{0} r^{3}},
$$

where $k$ is a wavenumber, $\varepsilon_{0}$ is the vacuum permittivity, $\boldsymbol{n}$ is the unit vector of $\boldsymbol{r} / r, \boldsymbol{\mu}$ is a dipole moment of the source placed at the origin, and $\omega$ is a frequency of the oscillation, where $\omega=k c$ with $c$ being the velocity of light. Aniline interacts with the electric field caused by the dipole $\boldsymbol{\mu}=(100,0,0)$ a.u. that is set above the molecule along the $y$ axis (aniline's principal axis), as shown in Fig. 2(c), where

a

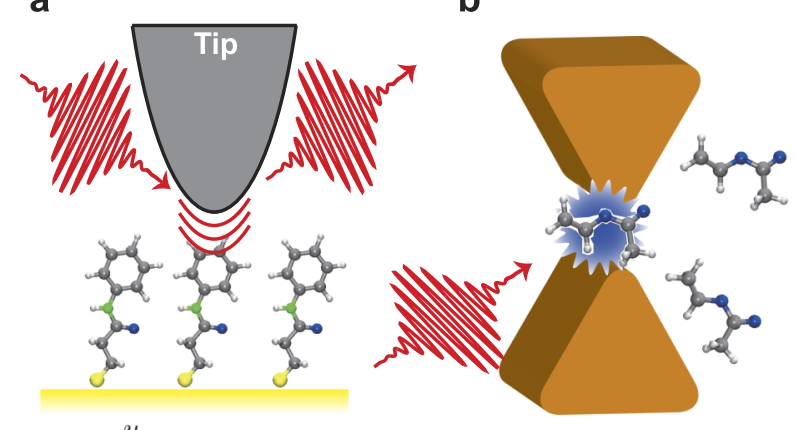

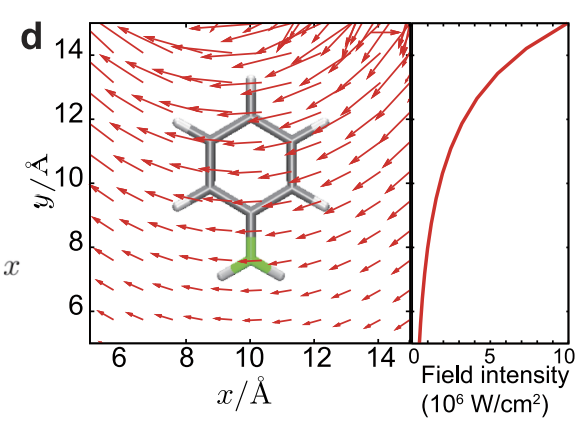

FIG. 2. (a) Schematic of a setup of scanning near-field infrared microscopy (SNIM), where incident light induces near-field around a tip, and (b) surface enhanced infrared absorption spectroscopy (SEIRAS) where an incident light induces enhanced near-field at the nano-gap between two nanostructures interacting with molecule. (c) The present computational model where nearfield is modeled by a dipole field. (d) Electric field lines (left) and intensity (right) along the cross section at $x=10 \AA$. $|\boldsymbol{\mu}|=100$ a.u. is chosen so that $\partial A / \partial b_{i}$ does not become very small. The field distribution is schematically shown in Fig. 2(c), and the actual field line and intensity gradient over the molecule are shown in Fig. 2(d) where the field mainly has $x$ component with weak but non-negligible $y$ component. The field intensity is very sharp, which is proportional to $r^{-6}$, according to Eq. (16). We should also comment on the validation of the present model. We consider that the use of the dipole radiation would be appropriate when the tip-molecule distance is larger than the tip radius, $(\sim 10 \mathrm{~nm})$, because the computed fields around a conical tip look like the dipole field. ${ }^{24,57}$ Actual comparisons will be made in the future study after combining the computational electrodynamics with the present methods using geometrically realistic metal tips. This near-field is used to calculate the effective electric field by substituting the $\boldsymbol{E}$ in Eq. (14) by this $\boldsymbol{E}_{\text {dip }}$ together with the following considerations.

The wavenumber $(k)$ dependence of the dipole field $\boldsymbol{E}_{\mathrm{dip}}$ is negligible in the IR region $\left(0-4000 \mathrm{~cm}^{-1}\right)$ due to the large difference in the molecular region $(r \sim 1 \mathrm{~nm})$ and wavelength $(\sim 1000 \mathrm{~nm})$, which leads to $e^{i k r} \sim 1$ for the wavelength $\lambda=2 \pi k$. From these results, we can use the right hand side of Eq. (16) for every wavelength of the present computations. As is apparent from Fig. 2(d), the near-field over the molecule looks mainly in the $x$ direction but has a complicated electric field lines, and also has the intensity gradient.

It would be worth to mention that the present model does not include the retardation effect because we consider the very small molecule in the close proximity to the photon source. For larger systems, however, the so-called retardation effect may appear non-negligible as such electric field at one end could be different to that at the other end of the large molecule. The retardation effect can be treated by considering the time-dependence of the electric field. To do that, the longitudinal and transverse parts of the nearfield should be evaluated separately because the longitudinal interaction is instantaneous, whereas the transverse one is retarded in the Coulomb gauge. ${ }^{47,48}$ Also the self-consistent effect is also neglected. For rigorous treatment, excited molecule should also affect the light source thus requiring the Maxwell equations to be solved taking into account the excited molecule. This is computationally demanding and in the present study, we assume that this self-consistent effect is small because of the small molecule.

\section{IR spectra using near-field vs uniform field}

Figure 3(a) shows the normal IR spectrum obtained by using the dipole moment of the molecule instead of $A$ in Eq. (5), whereas Figs. 3(b) and 3(c) show the IR absorption spectra for a uniform electric field in the $x$ direction $E_{x}$ and for the near-field, respectively, in the range $0-2000 \mathrm{~cm}^{-1}$. The normal IR spectrum corresponds to the IR absorption by randomly distributed molecules that are small compared to IR wavelength. In this case, the dipole approximation is valid and the excitation is considered isotropic, in other words, the vibrational modes accompanying atomic motions in every direction can be excited as long as the dipole moment changes by the vibration. On the other hand, IR spectra for Figs. 3(b) 


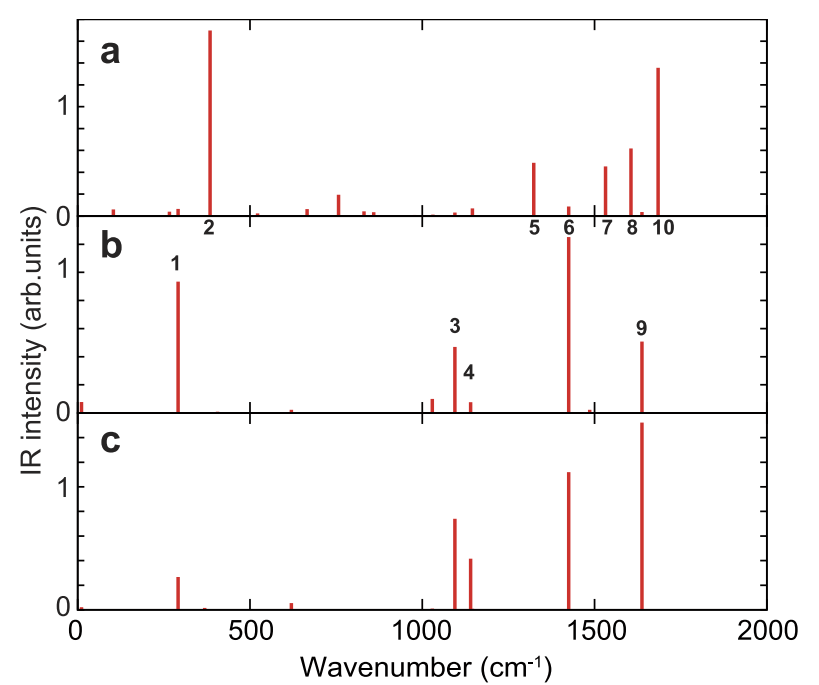

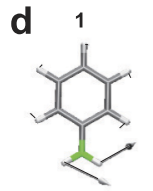
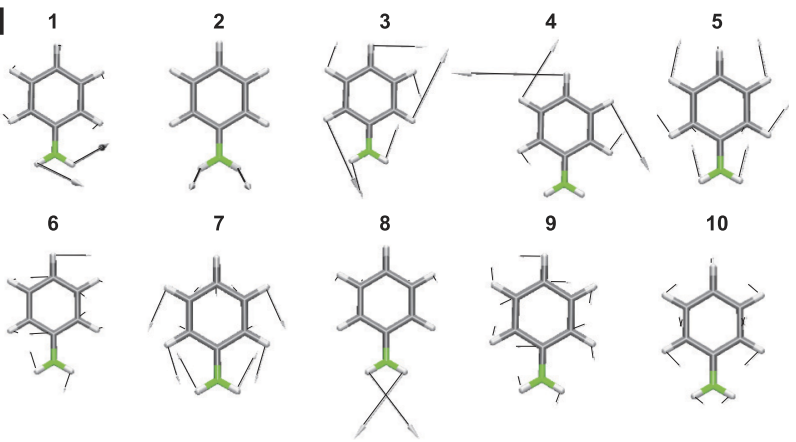

FIG. 3. IR spectra obtained by applying (a) isotropic uniform electric field, (b) anisotropic uniform electric field in the $x$ direction (i.e., the normal to the $C_{\mathrm{s}}$ axis), (c) the near-field, and (d) representative vibrational modes.

and 3(c) are obtained by applying the electric fields that are mainly in $x$ direction and are symmetric with respect to the molecular principal axis ( $y$-axis), thus only vibrational modes that are unsymmetric with respect to the $x$ direction. The vibrational modes that are symmetric with respect to the $x$ direction are forbidden, and of course the vibrations only with the $y$ and $z$ components are not excited. We use the uniform field to compare the results with the near-field, whose field distributions are shown in Figs. 2(c) and 2(d). Spectral differences between the uniform vs near-field come from intensity gradient, as well as the $y$ component of the nearfield. Figure 3(d) shows 10 representative vibrational modes. Figure 4 shows the same as Fig. 3 but for $3000-4000 \mathrm{~cm}^{-1}$. It should be noted that these IR peak intensities are normalized in each spectrum in terms of $\partial A / \partial b_{i}$ and thus the comparisons in intensity is only meaningful within the same spectrum (i.e, intensities for (a) and (b) are not directly comparable). Since the IR absorption spectra are very different for various applied fields, the inclusion of the field distribution is mandatory and our method will be valuable, especially for analyzing SNIM or SEIRAS experiments.

The symmetric vibrational modes with respect to the $C_{\mathrm{s}}$ axis disappear in the uniform and near-field due to the symmetry. Those peaks would be active for near-field if the tip is slightly displaced from the symmetry axis when scanning. The 1st peak is not very active for the IR but active for uniform and near-field excitations, but the relative

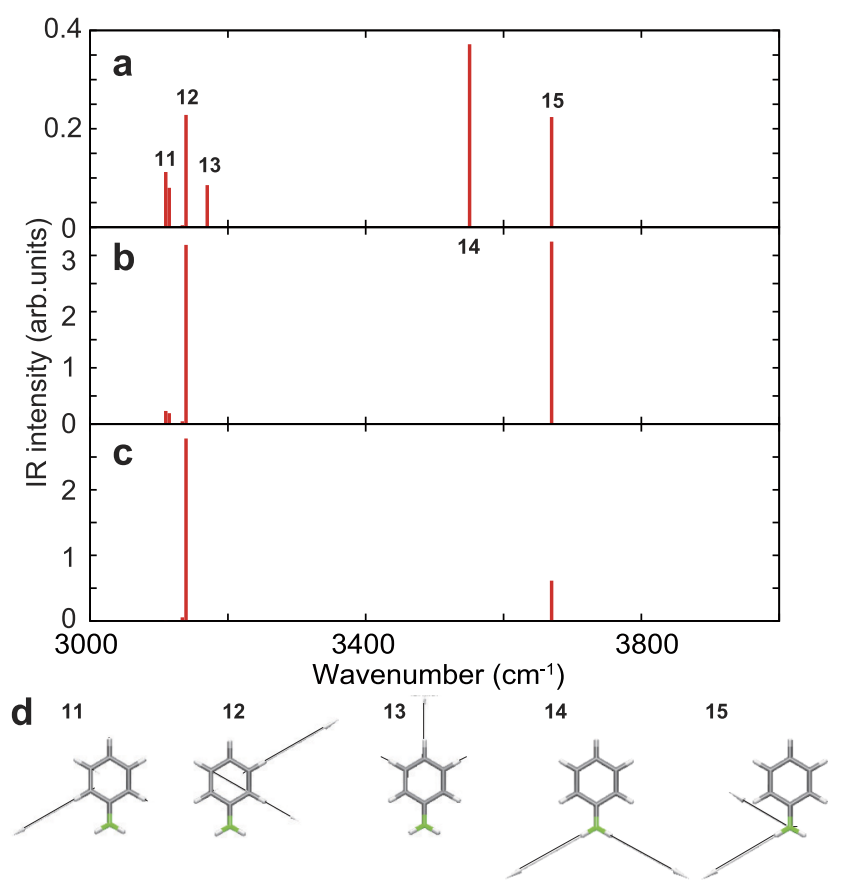

FIG. 4. The same as Fig. 3 but in the range $3000-4000 \mathrm{~cm}^{-1}$.

intensity for the near-field excitation is weak compared to the uniform excitation because the dominant contribution to this vibrational mode is the $\mathrm{NH}_{2}$ group which is far from the near-field source. Focusing on the vibrational modes of 3 and 4 in Figs. 3(b) and 3(c), the active group in the vibrational mode 4 is closer to the tip (near-field source) and therefore the relative intensity is enhanced for the near-field excitation due to the intensity gradient.

Next, let us look at the sensitivity to the intensity gradient. Figure 5 shows the IR spectra by changing the distance between the tip position and the molecular center. The most prominent tip-molecule distance dependence can be seen for the 4 th peak. The relative peak intensity of 4 shows the stronger distance dependence compared to 3 because the atomic motions in the normal mode 4 are localized to the $\mathrm{C}-\mathrm{H}$ groups close to the tip, while those in the normal mode 3 are delocalized over the molecule as shown in Fig. 3(d). The spectrum is almost the same beyond the distance larger than $500 \AA$ and indistinguishable beyond $1000 \AA$ as shown in Figs. 5(e) and 5(f). Thus, by changing the tip-molecule distance, one can perceive the extent of localization of the atomic motions for each vibration. The relative orientation of the molecule at the surface can also be studied as in the case of, e.g., IRRAS or SEIRAS because the surface selection rule coming from the anisotropic uniform electric field along the surface normal. Together with the field orientation, using the existence of field gradient, one might infer which end group orients to the surface from the relative intensity of the end groups like $\mathrm{NH}_{2}$ and $\mathrm{CH}$ in the present case.

Figure 6 shows the root-mean-square (RMS) obtained by the following equation:

$$
\mathrm{RMS} \equiv \sqrt{\frac{1}{N} \sum_{i}^{N}\left(I_{i}^{\mathrm{nf}}-I_{i}^{\mathrm{uni}}\right)^{2}}
$$




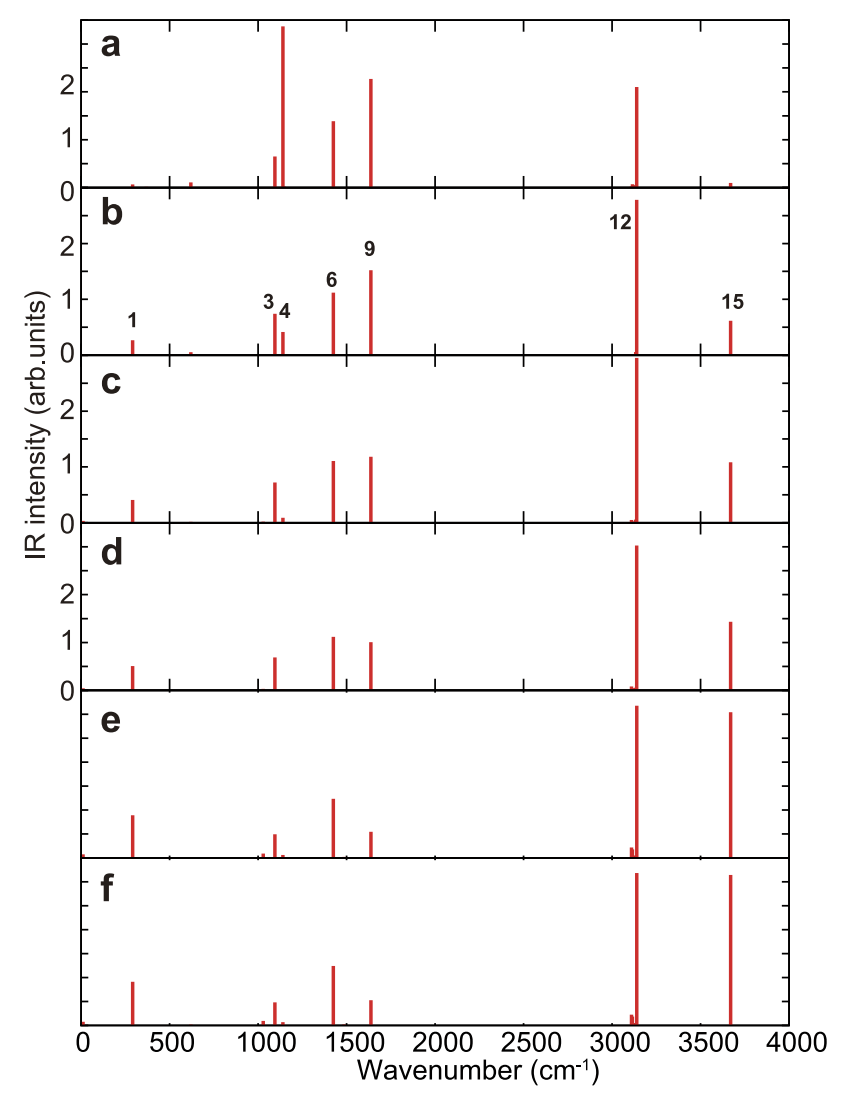

FIG. 5. IR spectra for near-field from the tip, where the distance between the tip and the molecular center are (a) 10, (b) 20, (c) 30, (d) 40, (e) 500, and (f) $1000 \AA$ A.

where $N, I_{i}^{\text {nf }}$, and $I_{i}^{\text {uni }}$ are the total number of the vibrational modes, $i$ th IR peak intensity of near-field absorption at each tip-molecule distance, and that obtained by anisotropic uniform field. These IR intensities are now normalized in each spectrum so as the largest peak to be 1 . The difference between the IR spectra with the uniform and near fields becomes very small when the tip-molecule distance is $500 \AA$, where slight deviation is found in the 15th peak shown in Fig. 4 because the $\mathrm{NH}_{2}$ group is apart from the tip and hence most affected by

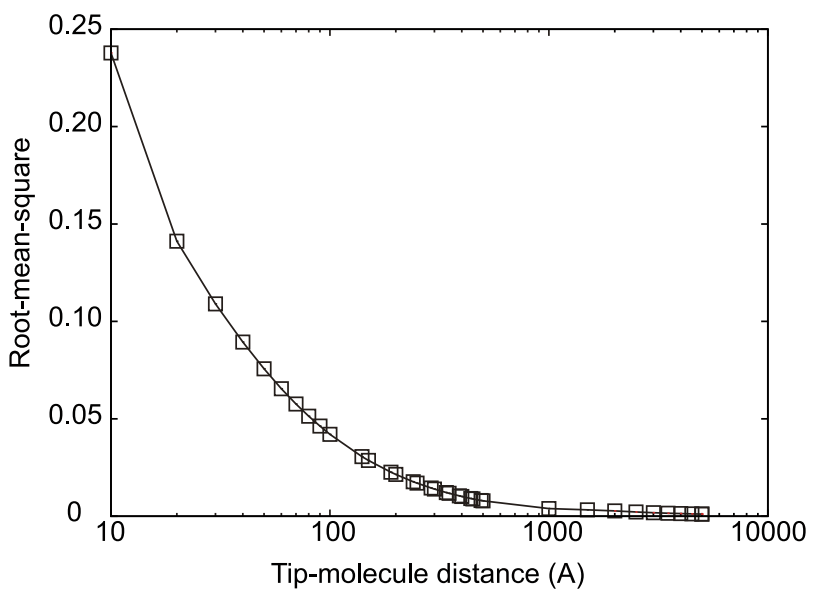

FIG. 6. Root-mean-square between the IR spectra obtained by using nearfield and anisotropic uniform field, plotted vs tip-molecule distance. the field gradient. Beyond the distance of $1000 \AA$, the spectral differences are negligible. In this range of the tip-molecule distance, the electric field is safely approximated by only the near part of the dipole radiation since the wavelength is larger than $25000 \AA\left(4000 \mathrm{~cm}^{-1}\right)$.

\section{SUMMARY}

The theoretical method to describe the interaction between molecular vibrations and arbitral electric fields and its computational scheme have been developed and applications have been presented. The multipolar Hamiltonian allows us to take into account the spatial distribution of electric fields by considering the original polarization operator without any approximations. The demonstrations of the present method are given for IRRAS and SNIM/SEIRAS in the model systems. These results successfully reflect the spatial structure of electric fields and molecular vibrational modes. For near-field excitations, two effects are important such as electric field lines and intensity gradients. By considering these effects, one can infer the molecular orientation at the surface. Although the present study uses very simple model electric fields as demonstrations, the method can be generally applicable to arbitrary nonuniform electric fields, thus combining two communities of the electronic structures and the optics. In the future, more realistic near-fields around nanostructures will be considered by computing the near-field by solving the Maxwell equations for combining the optics and molecular science fields.

\section{ACKNOWLEDGMENTS}

T.I. thanks the financial support for JSPS KAKENHI Grant No. 25810008. The computations were partly performed using Research Center for Computational Science, Okazaki, Japan.

\section{APPENDIX: DERIVATION OF EQ. (8)}

As in the previous study, ${ }^{45}$ the electron part becomes

$$
\begin{aligned}
\int\left\langle\hat{\boldsymbol{P}}_{\mathrm{elec}}\left(\boldsymbol{r}^{\prime}\right)\right\rangle \cdot \boldsymbol{E}\left(\boldsymbol{r}^{\prime}\right) d \boldsymbol{r}^{\prime} \\
=\int d \boldsymbol{r} d \boldsymbol{r}^{\prime} \Psi^{*}(\boldsymbol{r}) \hat{\boldsymbol{P}}_{\mathrm{elec}}\left(\boldsymbol{r}^{\prime}\right) \Psi(\boldsymbol{r}) \cdot \boldsymbol{E}\left(\boldsymbol{r}^{\prime}\right) \\
=-\int d \boldsymbol{r} d \boldsymbol{r}^{\prime} \Psi^{*}(\boldsymbol{r})(\boldsymbol{r}-\boldsymbol{R}) \\
\quad \times \int_{0}^{1} d \lambda \delta\left(\boldsymbol{r}^{\prime}-\boldsymbol{R}-\lambda(\boldsymbol{r}-\boldsymbol{R})\right) \Psi(\boldsymbol{r}) \cdot \boldsymbol{E}\left(\boldsymbol{r}^{\prime}\right) \\
=-\int d \boldsymbol{r}\left[\Psi^{*}(\boldsymbol{r}) \Psi(\boldsymbol{r})\right](\boldsymbol{r}-\boldsymbol{R}) \int_{0}^{1} d \lambda \\
\quad \times \int d \boldsymbol{r}^{\prime} \delta\left(\boldsymbol{r}^{\prime}-\boldsymbol{R}-\lambda(\boldsymbol{r}-\boldsymbol{R})\right) \boldsymbol{E}\left(\boldsymbol{r}^{\prime}\right) \\
\equiv-\int d \boldsymbol{r} \rho(\boldsymbol{r})(\boldsymbol{r}-\boldsymbol{R}) \cdot \int_{0}^{1} d \lambda \boldsymbol{E}(\boldsymbol{R}+\lambda(\boldsymbol{r}-\boldsymbol{R})) \\
\equiv-\int d \boldsymbol{r} \rho(\boldsymbol{r})(\boldsymbol{r}-\boldsymbol{R}) \cdot \boldsymbol{E}_{\mathrm{eff}}(\boldsymbol{r}),
\end{aligned}
$$


where $\Psi$ is the ground state wavefunction of the molecule and the electron density $\rho(\boldsymbol{r}) \equiv \Psi^{*}(\boldsymbol{r}) \Psi(\boldsymbol{r})$. The nuclear part is given as

$$
\begin{aligned}
\int d \boldsymbol{r}\left\langle\hat{\boldsymbol{P}}_{\mathrm{N}}(\boldsymbol{r})\right\rangle \cdot \boldsymbol{E}(\boldsymbol{r}) & =\int d \boldsymbol{r}\left\langle\sum_{\alpha} Z_{\alpha}\left(\hat{\boldsymbol{R}}_{\alpha}-\boldsymbol{R}\right) \int_{0}^{1} d \lambda \delta\left(\boldsymbol{r}-\boldsymbol{R}-\lambda\left(\hat{\boldsymbol{R}}_{\alpha}-\boldsymbol{R}\right)\right)\right\rangle \cdot \boldsymbol{E}(\boldsymbol{r}) \\
& =\sum_{\alpha} Z_{\alpha}\left(\boldsymbol{R}_{\alpha}-\boldsymbol{R}\right) \int_{0}^{1} d \lambda \int d \boldsymbol{r} \delta\left(\boldsymbol{r}-\boldsymbol{R}-\lambda\left(\boldsymbol{R}_{\alpha}-\boldsymbol{R}\right)\right) \cdot \boldsymbol{E}(\boldsymbol{r}) \\
& =\sum_{\alpha} Z_{\alpha}\left(\boldsymbol{R}_{\alpha}-\boldsymbol{R}\right) \int_{0}^{1} d \lambda \boldsymbol{E}\left(\boldsymbol{R}+\lambda\left(\boldsymbol{R}_{\alpha}-\boldsymbol{R}\right)\right) \\
& =\sum_{\alpha} Z_{\alpha}\left(\boldsymbol{R}_{\alpha}-\boldsymbol{R}\right) \cdot \boldsymbol{E}_{\mathrm{eff}}\left(\boldsymbol{R}_{\alpha}\right) .
\end{aligned}
$$

${ }^{1}$ P. C. Cross, E. B. Wilson, Jr., and J. C. Decius, Molecular Vibrations: The Theory of Infrared and Raman Vibrational Spectra (Dover Publications, Inc., NY, 1980).

${ }^{2}$ Y. Chabal, Surf. Sci. Rep. 8, 211 (1988).

${ }^{3}$ M. Diem, Introduction to Modern Vibrational Spectroscopy (Wiley, New York, 1993).

${ }^{4}$ Nano-Optics, edited by S. Kawata, M. Ohtsu, and M. Irie (Springer, Berlin, Heidelberg, 2002).

${ }^{5}$ F. Hoffmann, Surf. Sci. Rep. 3, 107 (1983).

${ }^{6}$ N. J. Harrick, Phys. Rev. Lett. 4, 224 (1960).

${ }^{7}$ N. J. Harrick, J. Phys. Chem. 64, 1110 (1960).

${ }^{8}$ J. Fahrenfort, Spectrochim. Acta 17, 698 (1961).

${ }^{9}$ S. Tojo, M. Hasuo, and T. Fujimoto, Phys. Rev. Lett. 92, 053001 (2004).

${ }^{10}$ S. Tojo and M. Hasuo, Phys. Rev. A 71, 012508 (2005).

${ }^{11}$ E. Smith and G. Dent, Modern Raman Spectroscopy: A Practical Approach (Wiley, England, 2005).

${ }^{12}$ H. M. Lee, S. M. Jin, H. M. Kim, and Y. D. Suh, Phys. Chem. Chem. Phys. 15, 5276 (2013).

${ }^{13}$ A. Hartstein, J. Kirtley, and J. C. Tsang, Phys. Rev. Lett. 45, 201 (1980).

${ }^{14}$ M. Osawa, Bull. Chem. Soc. Jpn. 70, 2861 (1997).

${ }^{15}$ F. Neubrech, A. Pucci, T. W. Cornelius, S. Karim, A. García-Etxarri, and J. Aizpurua, Phys. Rev. Lett. 101, 157403 (2008).

${ }^{16}$ P. Biagioni, J.-S. Huang, and B. Hecht, Rep. Prog. Phys. 75, 024402 (2012); e-print arXiv:1103.1568 (2011).

${ }^{17}$ B. Knoll and F. Keilmann, Nature 399, 134 (1999).

${ }^{18}$ R. Hillenbrand, T. Taubner, and F. Keilmann, Nature 418, 159 (2002).

${ }^{19}$ T. Taubner, R. Hillenbrand, and F. Keilmann, Appl. Phys. Lett. 85, 5064 (2004).

${ }^{20}$ M. B. Raschke, L. Molina, T. Elsaesser, D. H. Kim, W. Knoll, and K. Hinrichs, ChemPhysChem 6, 2197 (2005).

${ }^{21}$ E. Bründermann and M. Havenith, Annu. Rep. Prog. Chem., Sect. C: Phys. Chem. 104, 235 (2008).

${ }^{22}$ J. Steidtner and B. Pettinger, Phys. Rev. Lett. 100, 236101 (2008).

${ }^{23}$ R. Zhang, Y. Zhang, Z. C. Dong, S. Jiang, C. Zhang, L. G. Chen, L. Zhang, Y. Liao, J. Aizpurua, Y. Luo, J. L. Yang, and J. G. Hou, Nature 498, 82 (2013).

${ }^{24}$ L. Novotny and B. Hecht, Principles of Nano-Optics (Cambridge University Press, New York, 2006).

${ }^{25}$ J. K. Sass, H. Neff, M. Moskovits, and S. Holloway, J. Phys. Chem. 85, 621 (1981).

${ }^{26}$ E. J. Ayars, H. D. Hallen, and C. L. Jahncke, Phys. Rev. Lett. 85, 4180 (2000).

${ }^{27}$ P. K. Jain, D. Ghosh, R. Baer, E. Rabani, and A. P. Alivisatos, Proc. Natl. Acad. Sci. U. S. A. 109, 8016 (2012).

${ }^{28}$ M. Takase, H. Ajiki, Y. Mizumoto, K. Komeda, M. Nara, H. Nabika, S. Yasuda, H. Ishihara, and K. Murakoshi, Nat. Photonics 7, 550 (2013).
${ }^{29}$ D. V. Chulhai and L. Jensen, J. Phys. Chem. C 117, 19622 (2013).

${ }^{30}$ K. Cho, Prog. Theor. Phys. Suppl. 106, 225 (1991).

${ }^{31}$ J. K. Jenkins and S. Mukamel, J. Chem. Phys. 98, 7046 (1993).

${ }^{32}$ O. Keller, Phys. Rep. 268, 85 (1996).

${ }^{33}$ H. Ishihara, J. Phys.: Condens. Matter 16, R247 (2004).

${ }^{34}$ D. W. Brandl, N. A. Mirin, and P. Nordlander, J. Phys. Chem. B 110, 12302 (2006).

${ }^{35}$ J. Zhao, A. O. Pinchuk, J. M. McMahon, S. Li, L. K. Ausman, A. L. Atkinson, and G. C. Schatz, Acc. Chem. Res. 41, 1710 (2008).

${ }^{36} \mathrm{D}$. Abramavicius and S. Mukamel, J. Chem. Phys. 124, 034113 (2006).

${ }^{37}$ K. Lopata, D. Neuhauser, and R. Baer, J. Chem. Phys. 127, 154714 (2007).

${ }^{38}$ E. Lorin, S. Chelkowski, and A. Bandrauk, Comput. Phys. Commun. 177, 908 (2007).

${ }^{39}$ J. Y. Yan, W. Zhang, S. Q. Duan, X. G. Zhao, and A. O. Govorov, Phys. Rev. B 77, 165301 (2008).

${ }^{40}$ K. Lopata and D. Neuhauser, J. Chem. Phys. 130, 104707 (2009).

${ }^{41}$ T. Iida and H. Ishihara, Phys. Status Solidi A 206, 980 (2009).

${ }^{42}$ M. Yamaguchi, K. Nobusada, T. Kawazoe, and T. Yatsui, Appl. Phys. Lett. 106, 191103 (2015).

${ }^{43}$ L. Jensen, C. M. Aikens, and G. C. Schatz, Chem. Soc. Rev. 37, 1061 (2008).

${ }^{44}$ Y. S. Yamamoto, Y. Ozaki, and T. Itoh, J. Photochem. Photobiol., C 21, 81 (2014).

${ }^{45}$ T. Iwasa and K. Nobusada, Phys. Rev. A 80, 043409 (2009).

${ }^{46}$ T. Iwasa, K. Horiuchi, M. Shikishima, Y. Noguchi, S. Nagaoka, and A. Nakajima, J. Phys. Chem. C 115, 16574 (2011).

${ }^{47}$ C. Cohen-Tannoudji, J. Dupont-Roc, and G. Grynberg, Photons and Atoms: Introduction to Quantum Electrodynamics (Wiley-Interscience, 1989).

${ }^{48}$ D. P. Craig and T. Thirunamachandran, Molecular Quantum Electrodynamics (Dover Publications, 1998).

${ }^{49}$ S. Mukamel, Principles of Nonlinear Optical Spectroscopy, Oxford Series on Optical and Imaging Sciences (Oxford University Press, 1999).

${ }^{50}$ J. M. Soler, E. Artacho, J. D. Gale, A. García, J. Junquera, P. Ordejón, and D. Sánchez-Portal, J. Phys.: Condens. Matter 14, 2745 (2002); e-print arXiv:cond-mat/0111138 [cond-mat.mtrl-sci].

${ }^{51}$ J. P. Perdew, K. Burke, and M. Ernzerhof, Phys. Rev. Lett. 77, 3865 (1996).

${ }^{52}$ J. Junquera, Ó. Paz, D. Sánchez-Portal, and E. Artacho, Phys. Rev. B 64, 235111 (2001).

${ }^{53}$ C. J. Hirschmugl, P. Dumas, Y. J. Chabal, F. M. Hoffmann, M. Suhren, and G. P. Williams, J. Electron Spectrosc. Relat. Phenom. 64-65, 67 (1993).

${ }^{54}$ C. M. Kim, C.-W. Yi, and D. W. Goodman, J. Phys. Chem. B 106, 7065 (2002).

${ }^{55}$ M. Lust, A. Pucci, and A. Otto, J. Raman Spectrosc. 37, 166 (2006).

${ }^{56}$ J. D. Jackson, Classical Electrodynamics, 3rd ed. (Wiley, 1998).

${ }^{57}$ L. V. Brown, X. Yang, K. Zhao, B. Y. Zheng, P. Nordlander, and N. J. Halas, Nano Lett. 15, 1272 (2015). 